\title{
Galaxies Discovered Behind the Milky Way by the Dwingeloo Obscured Galaxies Survey
}

\author{
P. A. Henning \\ Institute for Astrophysics, University of New Mexico \\ 800 Yale Blvd., NE, Albuquerque, NM 87131 \\ R. C. Kraan-Korteweg \\ Observatoire de Paris-Meudon, D.A.E.C. \\ 92195 Meudon Cedex, France \\ and \\ Astronomy Department, University of Guanajuato \\ Apartado Postal 144, Guanajuato, GTO 36000, Mexico \\ A. J. Rivers \\ Institute for Astrophysics, University of New Mexico \\ 800 Yale Blvd., NE, Albuquerque, NM 87131 \\ A. J. Loan \\ Institute of Astronomy, University of Cambridge \\ Madingley Road, Cambridge CB3 0HA, UK \\ O. Lahav \\ Institute of Astronomy, University of Cambridge \\ Madingley Road, Cambridge CB3 0HA, UK \\ and \\ Anglo Australian Observatory \\ P.O. Box 296, Epping, NSW 2121, Australia \\ W. B. Burton \\ Leiden Observatory \\ Postbus 9513, NL-2300 RA Leiden, The Netherlands
}


Abstract

Our Galaxy blocks a significant portion of the extragalactic sky from view, hampering studies of large-scale structure. This produces an incomplete knowledge of the distribution of galaxies, and, assuming galaxies trace mass, of the gravity field. Further, just one unrecognized, nearby massive galaxy could have large influence over the Milky Way's motion with respect to the Cosmic Microwave Background.

Diligent surveys in the optical and infrared wavebands can find galaxies through moderate Galactic gas and dust, but close to the Galactic Plane, only radio surveys are effective. The entire northern Zone of Avoidance is being searched at $21 \mathrm{~cm}$ for galaxies using the Dwingeloo 25-m telescope. A shallow search for nearby, and/or massive galaxies has been completed, yielding five objects. Two of these galaxies were previously unknown, and although they are not likely members of the Local Group, are part of the nearby Universe. A deeper search continues, which will produce a flux-limited catalog of hidden galaxies. This portion of the survey is one-third complete, and has detected about 40 objects to date. Based on present understanding of the HI mass function, the complete survey should uncover 50 - 100 galaxies.

\section{Introduction}

The dust and high star density of the Milky Way galaxy obscures about $25 \%$ of the optical extragalactic sky, and about $10 \%$ in the infrared, producing a Zone of Avoidance (ZOA). The resulting incomplete coverage of surveys of external galaxies compromises studies of large-scale structures. The connectivity of superclusters and voids across the ZOA can be at present only poorly studied. Also, the all-sky distribution of mass which produces the observed dipole in the Cosmic Microwave Background has not been fairly mapped, and, assuming galaxies trace mass, filling in the missing information should produce a fairer representation of the gravity field. In particular, unrecognized nearby galaxies could have large influence, as the nearest galaxies may generate a sizable fraction of the dipole moment (KraanKorteweg 1989).

Careful searches in the optical and infrared wavebands can narrow the ZOA (see the many contributions in Unveiling Large-Scale Structures behind the Milky Way 1994), but in the regions of highest obscuration and infrared confusion near the Galactic Plane, only radio surveys can find galaxies. The 21-cm line of neutral hydrogen passes through the obscuration readily, so galaxies with sufficient HI can be found through detection of their 21-cm emission. Of course, this method will miss HI-poor, early-type galaxies. The only ZOA which remains at $21 \mathrm{~cm}$ for HI-rich objects comes from the inability 
to discriminate extragalactic sources with redshifts near zero velocity from Galactic HI. Until recently, radio receivers were not sensitive enough for observers to contemplate a full survey of the ZOA. The first attempt to find hidden galaxies was a pilot survey with the late 300-ft telescope, covering only $1.5 \%$ of the ZOA visible from Green Bank (Kerr \& Henning 1987; Henning 1992). This survey proved the efficacy of the method, uncovering 19 previously unknown objects, but the small beamsize of the 300-ft (and its subsequent demise) made a full survey with that instrument unfeasible.

To begin filling in the gaps in our knowledge of the distribution of optically-hidden galaxies, a full survey of the northern ZOA is being conducted with the 25-m radio telescope of the Netherlands Foundation for Research in Astronomy, in Dwingeloo. We report here on the results of a shallow, quick search (RMS noise per channel, $\sigma_{\mathrm{ch}}=175 \mathrm{mJy}$ ) for nearby, massive galaxies, and on the status of a deeper search $\left(\sigma_{\mathrm{ch}}=40 \mathrm{mJy}\right)$ for spirals to a redshift of $4000 \mathrm{~km} \mathrm{~s}^{-1}$. The two surveys together are referred to as the Dwingeloo Obscured Galaxies Survey.

\section{Survey Strategy}

\section{A. The Shallow Search}

The first phase of the survey was conducted with the aim of finding nearby and/or very massive galaxies. Even a single nearby, unrecognized M31-type galaxy would have an important gravitational effect on the Milky Way. To uncover such a galaxy, the entire ZOA accessible to the Dwingeloo telescope was surveyed with 5-minute integration times within the redshift range $V_{\mathrm{LSR}}=0-4000 \mathrm{~km} \mathrm{~s}^{-1}$, i.e. approximately 15000 partially overlapping pointings, with $\mathrm{HPBW} 0^{\circ} .6$ within $30^{\circ} \leq l \leq 220^{\circ} ;|b| \leq 5^{\circ} .25$. Negative velocities were not searched, as the Leiden/Dwingeloo Galactic HI survey had recently covered the area, searching the range $-450 \leq V_{L S R} \leq 400 \mathrm{~km} \mathrm{~s}^{-1}$ (Hartmann 1994, Hartmann \& Burton 1997), albeit with higher RMS due to shorter integration time per pointing. The obscured-galaxies survey utilizes the DAS-1000 channel digital autocorrelator spectrometer developed by Bos (1989) over $20 \mathrm{MHz}$ bandwidth, producing a velocity resolution of $4 \mathrm{~km} \mathrm{~s}^{-1}$, quite sufficient to distinguish narrow-linewidth galaxies from monochromatic noise spikes. (See Hartmann 1994, and Hartmann \& Burton 1997 for further details of the telescope and equipment set-up, as used for both the Galactic survey and the obscured-galaxies survey.) The obscured-galaxies survey was conducted in total-power mode, with ON-OFF pairs created from blindsurvey pointings. The exact survey strategy evolved over the course of the quick survey. For the first portion, covering the upper third of the survey area, $\left(b>1{ }^{\circ} .4\right)$, lines of sight were observed almost randomly, using software which had been developed by Dap Hartmann for the Leiden/Dwingeloo 
Galactic HI survey. The order of pointings was chosen by an algorithm which determined the length of time a position was above the horizon, preferentially observing those positions which were visible for only short periods. While this was fine for frequency-switched spectra, as was the case for the Galactic survey, it produced non-optimal ON-OFF pairs for the obscured-galaxies survey, as pointings observed close in time were not necessarily spatially close, and vice versa. For the bulk of the survey $(-5.25 \leq b \leq 1.05)$, observations were grouped into blocks of five consecutive pointings at constant $b$, with $l$ increased by 0.4 increments. From the sequence of pointings $1,2,3,4,5$, ON-OFF pairs were created as follows: $1-3,2-4,3-5,4-1,5-2$. The resulting spectra proved to be quite satisfactory, and it is this strategy which also forms the basis of the deeper (one hour per pointing) survey which is currently being carried out, and described below. Using this method, a real galaxian signal will appear twice, once as a positive signal, and again as a negative one, referenced against two independent scans. These blocks of five scans are organized into a honeycomb pattern with $\Delta b=0.35$ and adjacent rows offset by $\Delta l=0.2$. Over most of the survey, the sensitivity obtained was 175 mJy channel-to-channel variation. The sensitivity to HI mass as a function of distance can be derived from the expression

$$
M_{\mathrm{HI}}=2.36 \times 10^{5} r^{2} \int S(V) \mathrm{d} V \mathrm{M}_{\odot},
$$

where $r$ is the distance in Mpc and $\int S(V) \mathrm{d} V$ is the integral over the line of the flux density in Jy $\mathrm{km} \mathrm{s}^{-1}$. From past experience with HI surveys (e.g. Henning 1992, Kraan-Korteweg et al. 1997) we note that a galaxy is detected if its profile height satisfies

$$
F_{\mathrm{ob}} \bar{S}>\nu_{\mathrm{ch}} \sigma_{\mathrm{ch}},
$$

where $\bar{S}$ is the mean flux density at $21 \mathrm{~cm}$ and $\nu_{\mathrm{ch}}$ defines the threshold for detection (e.g. $\left.\nu_{\mathrm{ch}}=2.5\right) . \quad F_{\mathrm{ob}}$ is the 'off-beam' fudge factor, taking into account that a galaxy may lie away from the center of the telescope beam in a blind search, and hence will have a reduced observed flux. For a Gaussian beam, $F_{\mathrm{ob}}=0.7$. The sensitivity of HI mass derived under these assumptions is shown in Figure 1. Note that other detection criteria are possible, e.g. in terms of the integrated flux (Schneider 1996).

The performance of the system was checked periodically by observing NGC 3359 as a calibration source. Of 14,725 planned pointings, 12647 have been observed, equivalent to $86 \%$ of the $2000 \mathrm{deg}^{2}$ of the total survey area. The latitude range $1.4 \leq b \leq 5.25$ was $72 \%$ completed, and the range $-5^{\circ} .25 \leq b \leq 1.05$ was $94 \%$ observed, the difference arising from our increased sophistication of observing method for the lower portion. Missed pointings 
were scattered evenly through the area, save two significant gaps of $10^{\circ} \times 1^{\circ}$ in $1, \mathrm{~b}$ (Fig. 2). In addition, about $10 \%$ of the scans were rendered useless by solar or manmade interference. A further $20 \%$ were compromised over a narrow velocity range $\left(V_{L S R}=1000 \pm 200 \mathrm{~km} \mathrm{~s}^{-1}\right)$ by fairly monochromatic interference. The shape of this spurious signal was often irritatingly galaxian. This significantly lowered sensitivity to galaxies at this redshift since we were swamped by hundreds of false "galaxies" at $1000 \mathrm{~km} \mathrm{~s}^{-1}$. However, we could search these scans successfully over the rest of the velocity band.

Data from the telescope were translated into standard FITS format, using software developed by Dap Hartmann, and the ON-OFF pairs created as above. Blocks of five ON-OFF spectra were presented as postscript files for visual inspection. When candidate signals were identified, adjacent pointings were examined since it was possible that a galaxy could be detected in more than one pointing. For scans containing potential galaxian signals, the FITS data were loaded into the Spectral Line Analysis Package (Staveley-Smith 1985) for further analysis.

\section{B. The Deep Search}

After the shallow blind survey was completed, the telescope began the deep phase of the survey, with the aim of producing a flux-limited catalog. The strategy was much the same, with blocks of five observations of five

minutes per pointing, but now these blocks are repeated twelve times to reach an effective integration time of one hour per pointing, and a sensitivity of $40 \mathrm{mJy}$. This is not simply an improvement of $\sqrt{12}$ since we also switched to a two-bit correlator sampling mode, further improving sensitivity. The resulting sensitivity to HI mass is shown in Fig 3. For example, for a linewidth of $100 \mathrm{~km} \mathrm{~s}^{-1}$ we are sensitive to galaxies with $5 \times 10^{8} \mathrm{~h}^{-2} \mathrm{M}_{\odot}$ of $\mathrm{HI}$ out to $10 \mathrm{~h}^{-1} \mathrm{Mpc}$ and $5 \times 10^{9} \mathrm{~h}^{-2} \mathrm{M}_{\odot}$ of $\mathrm{HI}$ out to the edge of the survey's velocity range, $40 \mathrm{~h}^{-1} \mathrm{Mpc}$.

The first area to be deeply surveyed was the region $125^{\circ} \leq l \leq 145^{\circ}$; $-5.25 \leq b \leq 5.25$, encompassing the crossing of the Supergalactic plane and the Galactic plane. This has been completed, and now the telescope is observing the rest of the northern ZOA, starting at $b=-5.25$ and observing strips of increasing latitude. At the time of writing (June 1997) the deep survey had been completed from $b=-5.25$ to $b=-2^{\circ} .1$.

\section{Survey Results}

\section{A. The Shallow Search}

The shallow survey detected five galaxies, three of which were known previously. The sources are listed in Table 1, with HI parameters as observed 
during the shallow search period of the Dwingeloo survey. The positions of each pointing where the galaxies were detected are given, along with the strength of the signal at each location. For the position where the signal is at its strongest, the basic parameters of the line are measured. The extinction was derived from the Galactic HI column density (Weaver \& Williams 1973) assuming the gas-to-dust ratio derived by Burstein and Heiles (1982). The profiles, shown in Figure $4 \mathrm{a}$ - e, are constructed from the sum of the scans where each galaxy was detected.

Two of the previously known galaxies were originally discovered in the infrared (Maffei 2: Maffei 1968; IRAS 05596+1451: identified as a galaxy by Lu et al. 1990). Both have faint optical counterparts visible on the Palomar Sky Survey prints, and are heavily obscured spirals. The association of the Dwingeloo-detected objects with these cataloged galaxies rests not only on the angular position, but also on the correspondence with previous redshift measurements (Maffei 2: e.g. Bottinelli et al. 1971, Bottinelli \& Gougenheim 1976, Lewis \& Davies 1973, Love 1972, Shostak \& Weliachew 1971, and others; IRAS 05596+1451: Lu et al. 1990.) In the case of Maffei 2 , previous 21-cm observations show that the profile extends to negative velocities. We do not recover all of its emission, since our search range covered only positive velocities. The other source known previously, [H92] 34 , was discovered through a pilot blind 21-cm survey of a small portion of the ZOA made with the NRAO 300-ft telescope (Henning 1992). HI synthesis observations conducted with the VLA confirmed the detection, and indicated a mass and a profile shape consistent with the source being a normal, but totally obscured, spiral.

The other two sources detected are previously unknown, hidden galaxies. The galaxy Dwingeloo 1, which we catalog here also as Dw138.5-0.1, has been the subject of much follow-up observation (Kraan-Korteweg et al. 1994, Loan et al. 1996, Burton et al. 1996, Kuno et al. 1996, Li et al. 1996, Tilanus \& Burton 1997). To summarize, it is a massive barred spiral, with rotation velocity of $130 \mathrm{~km} \mathrm{~s}^{-1}$ compared to $220 \mathrm{kms}^{-1}$ for the Milky Way, implying a dynamical mass within a radius where the rotation curve is flat of roughly one-third the mass of the Milky Way. The exact mass is inaccurately known primarily due to its uncertain distance $(\approx 3 \mathrm{Mpc})$. Its angular location and redshift place it within the IC342/Maffei group of galaxies. It has a dwarf companion, Dwingeloo 2, which was discovered through sensitive follow-up HI synthesis observations with the Westerbork Synthesis Radio Telescope (Burton et al. 1996). The identification of this small galaxy as a companion to Dwingeloo 1 rests on its angular and kinematic proximity. Also, Dwingeloo 2 shows signs of distortion, possibly due to interaction with its more massive companion. 
The other galaxy discovered through the shallow search, Dw095.0+1.0 is also likely to be nearby, with a recession velocity $V_{\mathrm{LSR}}=159 \mathrm{~km} \mathrm{~s}^{-1}$, or $V_{\mathrm{G}}=378 \mathrm{~km} \mathrm{~s}^{-1}$ [where $V_{\mathrm{G}}=V_{\mathrm{LSR}}+220 \cos (b) \sin (l)$ ]. However, this source cannot be very massive. Naively assuming a distance of $3 \mathrm{Mpc}$, the HI mass would be about $8 \times 10^{7} \mathrm{~h}^{-2} \mathrm{M}_{\odot}$, bearing in mind that it may not be at the center of the beam, and the true flux density may be somewhat higher. (Planned follow-up HI synthesis observations will pin down its location and flux density.) Its narrow profile (FWHM $=87 \mathrm{~km} \mathrm{~s}^{-1}$ ) supports the idea that it is a hidden, nearby dwarf. There is no optical counterpart visible, due to high obscuration. Likewise, there is no obvious corresponding extragalactic IRAS source, although this is not surprising as IRAS was not very sensitive to dwarfs (Bothun et al. 1989; Sauvage et al. 1990), and confusion due to Galactic IR sources often masks low-latitude galaxies found in HI (Henning 1995). Its angular position and recession velocity indicate that it is not likely a member of the Local Group. Plotting recession velocity versus distance from the solar apex as determined by Sandage (1986), it lies well outside the range of known and even suspected Local Group galaxies (see Fig 1 of van den Bergh 1994). It is also not likely a member of the IC342/Maffei/Dw1 group, since it lies $\approx 40^{\circ}$ from that group's center (Krismer et al. 1995). However, it is close in velocity to NGC 6946 at $\left(l, b, V_{\text {hel }}=95.7,11.7,49 \mathrm{~km} \mathrm{~s}^{-1}\right.$; the velocity quoted is the average of measurements by Dean \& Davies 1975, Gordon et al. 1968, Rogstad \& Shostak 1972, Rots 1980, Tacconi \& Young 1986, Tifft \& Cocke 1988.) Since truly isolated galaxies are rare, this raises the possibility that these galaxies may be part of a previously unidentified nearby group. Such a group should be uncovered by the Dwingeloo Deep Search when it reaches this latitude range (except for low velocity members whose HI signals may be lost in Galactic HI.) If this hypothetical group exists, it is offset from the Supergalactic Plane by about $40^{\circ}$, considerably more than any other known group in the local Universe.

With the completion of the Shallow Search of the entire northern ZOA for nearby, massive HI galaxies, we can say with some confidence that the most massive, nearby, previously unidentified galaxy detectable in $\mathrm{HI}$ in this search area is Dwingeloo 1. However, since the survey covered only about $80 \%$ of the originally planned area, it is possible galaxies could have gone undetected. From an HI mass function, we estimate four galaxies should lie in the full survey region (Section IV) so $(0.2) \times(4)=1$ galaxy may lie in the unsurveyed area. The chances of missing a large, nearby, and therefore, extended galaxy are low, because such an object would likely be detectable in several adjacent pointings, all of which would have to be missed for the galaxy to escape detection. Thus, it is fairly unlikely that there is another previously unidentified, massive galaxy whose gravitational influence impacts Local Group peculiar motion or internal dynamics in an important way in 
the area of the survey.

Finally, we consider the possibility of a large, unrecognized galaxy lying outside the range of our survey. From the distribution of Galactic HI (data from Weaver \& Williams 1973, map presented by Bloemen 1983) and the conversion from $\mathrm{HI}$ column density to extinction $\mathrm{A}_{\mathrm{B}}$, assuming a constant Galactic gas-to-dust ratio (Burstein \& Heiles 1978), we find the extinction falls to roughly 2.5 magnitudes at $|b|=5.25$, with some patches exceeding 4 magnitudes of obscuration. An $\mathrm{A}_{\mathrm{B}}$ of 2.5 magnitudes corresponds to a diminution in angular size of a factor of three (Cameron 1990). A galaxy near enough and large enough to have significant gravitational influence, an M31like galaxy, would still be recognizable through this amount of obscuration. Of course, we are truly blind to galaxies in the southern ZOA, longitude range from $1=220^{\circ}$ to $30^{\circ}$, which lies below the declination limit of the Dwingeloo telescope. Within the same latitude range as the northern search, this corresponds to $1800 \mathrm{deg}^{2}$, comparable to the $2000 \mathrm{deg}^{2}$ we surveyed in the north. Thus, about four galaxies would be detected if this southern area were surveyed with the search parameters used in this survey, as discussed in Section IV. A more sensitive 21-cm survey being conducted with the Parkes 64-m telescope will cover this southern gap (Staveley-Smith 1996). The remaining blind spot of any 21-cm survey for galaxies in the ZOA is the slice in velocity space contaminated by Galactic HI, within roughly $\pm 200 \mathrm{~km} \mathrm{~s}^{-1}$, with contamination level strongly dependent on 1 and b.

To double check that the Shallow Search did indeed recover the nearby known galaxies it should have, a search for all cataloged galaxies within our angular and velocity restrictions was conducted using the Lyon-Meudon Extragalactic Database (LEDA), and the NASA/IPAC Extragalactic Database (NED). This produced a list of five galaxies from other surveys with systemic velocities between $0-1000 \mathrm{~km} \mathrm{~s}^{-1}$. Of the five, we did detect two (Maffei 2, Dw136.5-0.3, and IRAS05596+1451, Dw194.1-3.8). The other three were not recovered for well understood reasons: Maffei 1, an elliptical, which would not be detected in an HI survey; [H92] 11, a galaxy discovered through an earlier HI survey (Henning 1992); and MB 1, an HI-deficient, late type spiral in the IC 342/Maffei/Dw1 group (McCall et al. 1995; Huchtmeier \& van Driel 1996). The flux densities of the latter two galaxies are well below the sensitivity limit of the Shallow Search, but they been observed and detected by the continuing Deep Search, described below.

\section{B. The Deep Search}

Here we present a progress report on the Deep Search phase of the Dwingeloo Obscured Galaxies Survey. We have detected 40 galaxies over the $790 \mathrm{deg}^{2}$ surveyed to date. Half of these galaxies lie in the region 
of the Galactic Plane/Supergalactic Plane crossing. The locations of the galaxies are shown in Fig. 5, along with the positions of previously cataloged objects in the search region and surrounding higher latitudes. A few sources await confirmation with the Dwingeloo telescope. A list of objects' positions and 21-cm flux densities will be published after HI synthesis followup observations are completed. With these data, we will investigate the properties of HI-selected galaxies in different environments (e.g. Local Void vs. Local Supercluster) and the observed HI mass function.

\section{Detection Predictions from an HI Mass Function}

Here we attempt to predict the number of HI galaxies expected to be found in a 21-cm survey, observed with the parameters being used for the Dwingeloo work. The prediction attempts to mimic the detection of galaxies by eye.

A prediction of the number of HI galaxies expected to be detected by a survey requires some knowledge of the HI mass function, $\phi\left(M_{\mathrm{HI}}\right)$. The complete HI mass function will be a bivariate function of the HI mass and the velocity width, $\phi\left(M_{\mathrm{HI}}, \Delta V\right)$. However, the present data sets are not sufficient to estimate the bivariate function, and (following Rao \& Briggs 1993 and Schneider 1996) the HI mass function is assumed to be approximated by a Schechter (1976) function for every $\Delta V$,

$$
\phi\left(M_{\mathrm{HI}}\right) \mathrm{d} M_{\mathrm{HI}}=\phi_{*}\left(\frac{M_{\mathrm{HI}}}{M_{*}}\right)^{-\alpha} \exp \left(\frac{-M_{\mathrm{HI}}}{M_{*}}\right) d\left(\frac{M_{\mathrm{HI}}}{M_{*}}\right),
$$

where $\alpha$ is the low-mass-end slope, $M_{*}$ is the characteristic HI mass, and $\phi_{*}$ is a normalization parameter. After Zwaan et al. (1997), we adopt $\alpha=1.25$, $M_{*}=0.4 \times 10^{10} \mathrm{M}_{\odot}, \phi_{*}=0.013$ galaxies per $(\mathrm{Mpc} / \mathrm{h})^{3}$.

The derivation of the HI mass function by Henning (1995), Rao \& Briggs (1993), Zwaan et al. (1997), and others uses the $1 / V_{\max }$ method. This method makes the assumption that the galaxy population is distributed in a homogeneous manner. However, galaxies are clumped together in groups and clusters, hence this method produces a biased statistic. By using a clustering-independent parametric maximum-likelihood technique, such as the STY technique (after Sandage et al. 1979), the HI mass function may be more fairly derived. This method has been used recently for an opticallyselected sample (Solanes et al. 1996.) Work is underway to construct HI mass functions in this manner for various HI-selected samples (Lahav et al. in prep.)

A. The Expected Number of Galaxies 
For a uniform distribution, the number of galaxies expected to be detected in a survey out to a distance $R_{\max }$ with solid angle $\Omega_{s}$ can be calculated by integrating over the mass function:

$$
N\left(<R_{\max }\right)=\Omega_{s} \int_{0}^{R_{\max }} \mathrm{d} r r^{2} \int_{x_{\min }}^{\infty} \phi(x) d x,
$$

where $x=M_{\mathrm{HI}} / M_{*}$, and the minimum HI mass to be observed (from Equation 1) is

$$
M_{\mathrm{HI}, \min }=x_{\min } M_{*}=2.36 \times 10^{5}\left(\frac{\Delta V \nu_{\mathrm{ch}} \sigma_{\mathrm{ch}}}{F_{\mathrm{ob}}}\right) r^{2} M_{\odot} .
$$

As before, $\sigma_{\mathrm{ch}}$ is the RMS noise per channel, $\nu_{\mathrm{ch}}$ defines the threshold for detection (e.g. $\nu_{\mathrm{ch}}=2.5$ ), and $F_{\mathrm{ob}}$ is the factor which takes into account that a galaxy may lie away from the center of the telescope beam in a blind search, and hence will have a reduced observed flux.

Assuming that the mass function is a Schechter function, then

$$
\int_{x_{\text {min }}}^{\infty} \phi(x) \mathrm{d} x=\phi_{*} \Gamma\left[1-\alpha, x_{\min }(r)\right]
$$

where $\Gamma$ is the incomplete Gamma function. Thus, the result depends on the combination of parameters $\left(\Delta V \nu_{\mathrm{ch}} \sigma_{\mathrm{ch}}\right) /\left(F_{\mathrm{ob}} M_{*}\right)$.

If the survey extends to infinity $\left(R_{\max }=\infty\right)$ then the integral in Equation 4 can be solved analytically:

$$
N(<\infty)=2.9 \times 10^{-9} \Omega_{s} \phi_{*} \Gamma\left(\frac{5}{2}-\alpha\right)\left(\frac{M_{*}}{M_{\odot}}\right)^{\frac{3}{2}}\left(\frac{\Delta V \nu_{\mathrm{ch}} \sigma_{\mathrm{ch}}}{F_{\mathrm{ob}}}\right)^{\frac{-3}{2}},
$$

where $\Gamma$ is the complete Gamma function; if $\alpha=1.25$ then $\Gamma((5 / 2)-\alpha)=$ 0.906 . This calculation extends to infinity, and thus the predicted number of galaxies will be higher than the number expected within a survey with a finite maximum depth. However, it does produce a fiducial number of galaxies. Once the Dwingeloo Deep Search is complete, this will provide an estimate for the number of galaxies that would have been detected if they did not lie beyond $R_{\max }$.

\section{B. Results and Predictions}

The appropriate parameters for the survey are: $R_{\max }=4000 \mathrm{~km} \mathrm{~s}^{-1}$, $\sigma_{\mathrm{ch}}=175 \mathrm{mJy}$ (for the Shallow Survey), $F_{\mathrm{ob}}=1, \nu_{\mathrm{ch}}=2.5$. Assuming 
a fiducial value of $\Delta V=185 \mathrm{~km} \mathrm{~s}^{-1}$ (the mean value found by Henning (1995) for an HI-selected sample) and the HI mass function parameters from Zwaan et al. (1997), then $N=8$ galaxies should be detected in the $2000 \mathrm{deg}^{2}$ survey area $\left(\Omega_{s}=0.6 \mathrm{str}\right)$. The number of expected galaxies is the same if $R_{\max }=\infty$ : galaxies cannot be detected beyond the survey limits with an integration time of only $5 \mathrm{~min}$. Remembering the off-beam factor, if $F_{\mathrm{ob}}=0.7$ then the predicted number in the Shallow Survey is $N=4$ galaxies. The observed number is five galaxies, very close to this prediction.

For the Deep Search portion of the survey $\left(\sigma_{\mathrm{ch}}=40 \mathrm{mJy}\right)$ the corresponding predictions are $N=62$ galaxies for $F_{\mathrm{ob}}=1.0$, and $N=39$ galaxies for $F_{\mathrm{ob}}=0.7$. However, these calculations take no account of density enhancements within the survey area. The area chosen for the survey includes regions that are known to be over-dense. For example, the Supergalactic Plane is 10-40 per cent denser than the uniform background (Lahav et al. in prep). Thus, the full Dwingeloo Deep Survey should be expected to find 50-100 galaxies. This is quite in line with the detection of 40 galaxies at this

stage of survey completion, $790 \mathrm{deg}^{2}$ observed of the $2000 \mathrm{deg}^{2}$ total area to be done.

\section{Summary and Future Directions}

A shallow search for nearby, and/or massive galaxies detectable at 21$\mathrm{cm}$ has been completed over the northern ZOA, with the detection of five galaxies. Two of these were previously unknown, and while their distances are not as yet very accurately determined, they are part of the local Universe. A more sensitive search continues, which should uncover 50 - 100 galaxies to the edge of the survey range, $R_{\max }=4000 \mathrm{~km} \mathrm{~s}^{-1}$.

The southern ZOA is currently being surveyed at 21-cm with the new multibeam receiver system on the Parkes radiotelescope (Staveley-Smith 1997). Extrapolating from observed HI mass functions based on only dozens of galaxies, as in Section IV, this survey should uncover thousands of galaxies, due to its excellent sensitivity and deep velocity coverage, $R_{\max }=12,200 \mathrm{~km} \mathrm{~s}^{-1}$. For the present Dwingeloo Survey, the galaxies are uncovered by visual inspection of the spectra. An alternative approach for handling future HI surveys, like the Parkes multibeam survey, is to attempt to devise an automated galaxy detection algorithm, e.g. Loan (1997).

\section{Acknowledgments}

We are grateful to D. Hartmann for the development of special-purpose software and for running a variety of tests which established the feasibility of the Dwingeloo Obscured Galaxies Survey; to G. Hau and H. Ferguson for 
their early work in getting the survey running routinely, to A. Foley and D. Moorrees for their supervision of the day-to-day telescope operations, and to D. Lynden-Bell for helpful discussions.

The research has made use of the NASA/IPAC Extragalactic Database (NED) which is operated by the Jet Propulsion Laboratory, California Institute of Technology, under contract with the National Aeronautics and Space Administration. We have also made use of the Lyon-Meudon Extragalactic Database (LEDA) supplied by the LEDA team at the CRALObservatoire de Lyon (France.) The Dwingeloo 25-m radiotelescope is supported by the Netherlands Foundation for Scientific Research (NWO).

The research of PH is supported by NSF Faculty Early Career Development (CAREER) Program award AST-9502268. The research of RCKK has been supported by an EC grant.

\section{References}

Bloemen, J.B.G.M. 1983, in Surveys of the Southern Galaxy, ApScS Library, vol. 105, eds. W.B. Burton \& F.P. Israel, Reidel: Dordrecht

Bos, A. 1989, The 1024 Channel Spectrometer for the Dwingeloo Telescope, Internal Technical Report, 188, NFRA

Bothun, G.D., Lonsdale, C.J., \& Rice, W. 1989, ApJ, 341, 129

Bottinelli, L., Chamaraux, P., Gerard, E., Gouguenheim, L, Heidmann, J., Kazes, I., \& Lauque, R. 1971, A\&A, 12, 264

Bottinelli, L., \& Gouguenheim, L. 1976, A\&A, 47, 381

Burstein, D., \& Heiles, C. 1978, ApJ, 225, 40

Burstein, D., \& Heiles, C. 1982, AJ, 87, 1165

Burton, W.B., Verheijen, M.A.W., Kraan-Korteweg, R.C., \& Henning, P.A. 1996, A\&A., 309, 687

Cameron, L.M. 1990, A\&A, 233, 16

de Vaucouleurs, G., de Vaucouleurs, A., \& Corwin, H.G. 1976, Second Reference Catalogue of Bright Galaxies (Austin: Univ. of Texas Press)

Dean, J.F., \& Davies, R.D. 1975, MNRAS, 170, 503

Gordon, K.J., Remage, N.H., \& Roberts, M.S. 1968, ApJ, 154, 845

Hartmann, D. 1994, Ph.D. Thesis, R.U. Leiden 
Hartmann, D., \& Burton, W.B. 1997, Atlas of Galactic Neutral Hydrogen, Cambridge University Press

Henning, P.A. 1992, ApJS, 78, 365

Henning, P.A. 1995, ApJ, 450, 578

Huchtmeier, W.K., \& van Driel, W. 1996, A\&A, 305, 25

Kerr, F.J., \& Henning, P.A. 1987, ApJ, 320, L99

Kraan-Korteweg, R.C. 1989, in Rev. in Modern Astron. 2, ed. G. Klare, Springer: Berlin

Kraan-Korteweg, R.C., Woudt, P.A., \& Henning, P.A. 1997, PASA, 14, 15

Kraan-Korteweg, R.C., Loan, A.J., Burton, W.B., Lahav, O., Ferguson, H.C., Henning, P.A., \& Lynden-Bell, D. 1994, Nature, 372, 77

Krismer, M., Tully, R.B., \& Gioia, I.M. 1995, AJ, 110, 1584

Kuno, N., Vila-Vilaro, B., \& Nishiyama, K. 1996, PASJ, 48, 19

Lewis, B.M., \& Davies, R.D. 1973, MNRAS, 165, 213

Li, J.G., Zhao, J.H., Ho, P.T.P., \& Sage, L.J. 1996, A\&A, 307, 424

Loan, A.J. 1997, Ph.D. Thesis, University of Cambridge

Loan, A.J., Maddox, S.J., Lahav, O., Balcells, M., Kraan-Korteweg, R.C., Assendorp, R., Almoznino, E., Brosch, N., Goldberg, E., \& Ofek, E.O. 1996, MNRAS, 280, 537

Love, R. 1972, Nature, 235, 53

Lu, N.Y., Dow, M.W., Houck, J.R., Salpeter, E.E., \& Lewis, B.M. 1990, ApJ, 357, 388

McCall, M.L., Buta, R.J., \& Huchtmeier, W.K. 1995, IAU Circular No. 6159

Maffei, P. 1968, PASP, 80, 618

Nilson, P. 1973, Uppsala General Catalogue of Galaxies, Uppsala Astron. Obs. Ann., 6

Rao, S., \& Briggs, F.H. 1993, ApJ, 419, 515

Rogstad, D.H., \& Shostak, G.S. 1972, ApJ, 176, 315

Rots, A.H. 1980, A\&AS, 41, 189 
Sandage, A. 1986, ApJ, 307, 1

Sandage, A., Tammann, G.A., \& Yahil, A. 1979, ApJ, 232, 352

Sauvage, M., Thuan, T.X., \& Vigroux, L. 1990, A\&A, 237, 296

Schechter, P. 1976, ApJ. 203, 297

Schneider, S.E. 1996, The Minnesota Lectures on Extragalactic Neutral Hydrogen, ASP Conf. Ser. 106, ed. E.D. Skillman, p. 323

Shostak, G.S., \& Weliachew, L 1971, ApJ, 169, L71

Solanes, J. M., Giovanelli, R., \& Haynes, M. P. 1996, ApJ, 461, 609

Staveley-Smith, L. 1985, Ph.D. Thesis, Univ. of Manchester

Staveley-Smith, L. 1997, PASA, 14, 111

Tacconi, L.J., \& Young, J.S. 1986, ApJ, 308, 600

Tifft, W.G., \& Cocke, W.J. 1988, ApJS, 67, 1

Tilanus, R.P.J., \& Burton, W.B. 1997 A\&A, in press

Unveiling Large-Scale Structures behind the Milky Way, eds. C. Balkowski and R.C. Kraan-Korteweg, 1994, ASP Conf. Ser. 67

van den Bergh, S. 1994, AJ, 107, 1328

Weaver, H.F., \& Williams, D.R.W. 1973, A\&AS, 8, 1

Zwaan, M., Briggs, F., \& Sprayberry, D. 1997, PASA, 14, 126 
Table 1. Galaxies Detected through the Dwingeloo Shallow Search

\begin{tabular}{|c|c|c|c|c|c|c|c|c|}
\hline Galaxy & $\begin{array}{c}1 \\
(\operatorname{deg})\end{array}$ & $\begin{array}{c}\mathrm{b} \\
(\mathrm{deg})\end{array}$ & $\begin{array}{c}\mathrm{V}_{\mathrm{LSR}} \\
\left(\mathrm{km} \mathrm{s}^{-1}\right)\end{array}$ & $\begin{array}{c}\Delta \mathrm{V}_{50} \\
\left(\mathrm{~km} \mathrm{~s}^{-1}\right)\end{array}$ & $\begin{array}{l}\text { Peak } \\
(\mathrm{Jy})\end{array}$ & $\begin{array}{c}\text { HI Flux } \\
\left(\mathrm{Jy} \mathrm{km} \mathrm{s}^{-1}\right)\end{array}$ & $\begin{array}{c}\mathrm{A}_{V} \\
(\mathrm{mag})\end{array}$ & Opt? \\
\hline Dw095.0+1.0 & $\begin{array}{l}95.0 \\
95.4\end{array}$ & $\begin{array}{l}1.1 \\
1.1\end{array}$ & 159 & 87 & $\begin{array}{l}0.60 \\
0.45\end{array}$ & 38 & 6.5 & $\mathrm{~N}$ \\
\hline $\begin{array}{l}{[\mathrm{H} 92] 34} \\
=\text { Dw095.1+1.6 }\end{array}$ & $\begin{array}{l}95.0 \\
95.2 \\
95.4\end{array}$ & $\begin{array}{l}1.8 \\
1.4 \\
1.8\end{array}$ & 1046 & 93 & $\begin{array}{l}0.96 \\
0.69 \\
0.59\end{array}$ & 77 & 6.5 & $\mathrm{~N}$ \\
\hline $\begin{array}{l}\text { Maffei } 2 \\
=\text { Dw136.5-0.3 }\end{array}$ & $\begin{array}{l}136.6 \\
136.4\end{array}$ & $\begin{array}{l}-0.4 \\
-0.7\end{array}$ & 98 & 84 & $\begin{array}{l}0.88 \\
0.37\end{array}$ & 53 & 4.3 & $Y$ \\
\hline $\begin{array}{l}\text { Dw1 } \\
=\text { Dw138.5-0.1 }\end{array}$ & $\begin{array}{l}138.6 \\
138.8 \\
138.2 \\
138.4\end{array}$ & $\begin{array}{c}-0.4 \\
0.0 \\
-0.4 \\
0.0\end{array}$ & 110 & 184 & $\begin{array}{c}1.31 \\
1.18 \\
1.08: \\
1.00\end{array}$ & 152 & 5.3 & $\mathrm{Y}$ \\
\hline $\begin{array}{l}\text { IRAS 05596+1451 } \\
=\text { Dw194.1-3.8 }\end{array}$ & $\begin{array}{l}194.2 \\
194.0 \\
194.4 \\
194.6\end{array}$ & $\begin{array}{l}-3.9 \\
-3.5 \\
-3.5 \\
-3.9\end{array}$ & 745 & 231 & $\begin{array}{l}0.87 \\
0.58 \\
0.56 \\
0.46\end{array}$ & 131 & 2.7 & $\mathrm{Y}$ \\
\hline
\end{tabular}

Note: For all galaxies except Dw095.0+1.0, optical or IR identification, or HI synthesis observations have provided accurate positions, which are reflected in the galaxies' names. For Dw095.0+1.0, the name is based on the search point where it shows the strongest signal, which is not necessarily the galaxy's true position.

The uncertainty in peak flux for the third position quoted for Dw138.5-0.1 is due to interference. 
Figure Captions

Figure 1. Sensitivity to HI mass as a function of distance for three representative linewidths for the Shallow Search, with integration time of 5 minutes per pointing. The channel-to-channel RMS variation achieved is $175 \mathrm{mJy}$, the threshold for detection is taken to be 2.5 times the RMS noise over the profile. Account is taken of the random placement of a galaxy in the Gaussian beam, which lowers the observed flux to an average of $70 \%$ of the beam-center value.

Figure 2. Coverage map of the Shallow Search. Over the latitude range $1.4 \leq b \leq 5.25$, the survey was $72 \%$ complete. From $-5^{\circ} .25 \leq b \leq 1.05$, $94 \%$ of the planned pointings were observed. About $10 \%$ of scans were badly disturbed by interference.

Figure 3. Sensitivity to HI mass as a function of distance for three representative linewidths for the Deep Search, with integration time of 1 hour per pointing. The channel-to-channel RMS variation achieved is $40 \mathrm{mJy}$, the threshold for detection is again taken to be 2.5 times the RMS noise over the profile. As in Fig. 1, account is taken of the random placement of a galaxy in the Gaussian beam.

Figure 4. HI profiles of the five galaxies detected by the Shallow Search. The profiles show the sum of emission for all pointings where 21-cm signal is detected for each galaxy.

Figure 5. Distribution of previously-cataloged galaxies with redshifts measured to be within $4000 \mathrm{~km} \mathrm{~s}^{-1}$ are shown as crosses, and the galaxies detected in Dwingeloo are shown as squares (Shallow Survey) and triangles (Deep Survey). The previously-cataloged galaxies mostly come from optical compilations [235 from the Uppsala General Catalog (Nilson 1973) and 62 from the Second Reference Catalogue of Bright Galaxies (de Vaucouleurs et al. 1976)]. The rest are from smaller optical surveys, or from IRAS. The dotted lines indicate the area of the sky surveyed to date by the Deep Survey. The Supergalactic Plane runs roughly vertically through the Galactic Plane, crossing in the longitude range $130^{\circ}-145^{\circ}$. Two Shallow Survey detections lie in the IC $342 /$ Maffei/Dw1 group at $l \approx 140^{\circ}, b \approx 0^{\circ}$. The Local Void is evident as a general underdensity of galaxies between $l \approx 30^{\circ}-75^{\circ}$. Several galaxies detected by the Deep Survey lie in this region. 


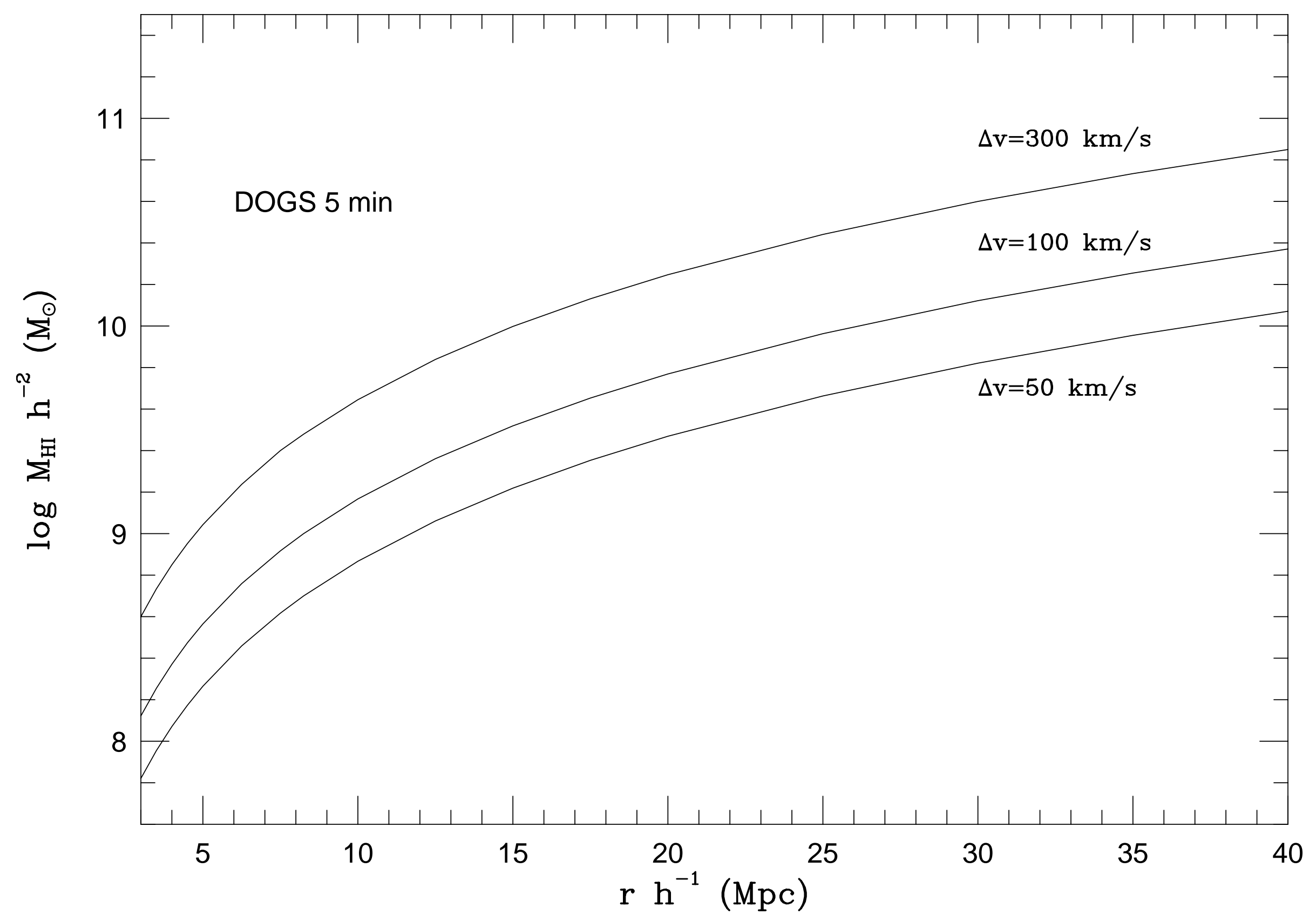


5min survey: $12647 / 14725$ pointings

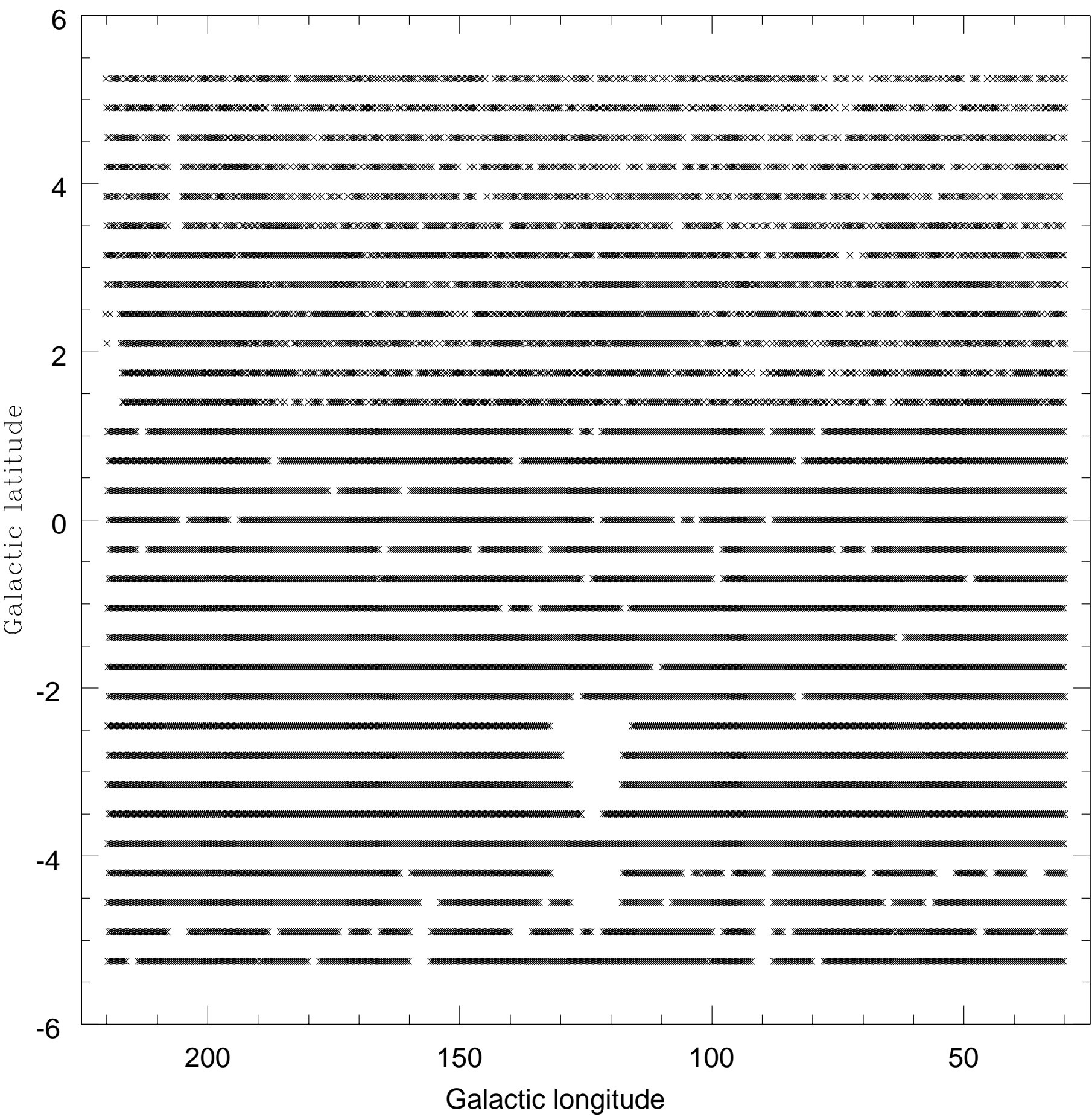




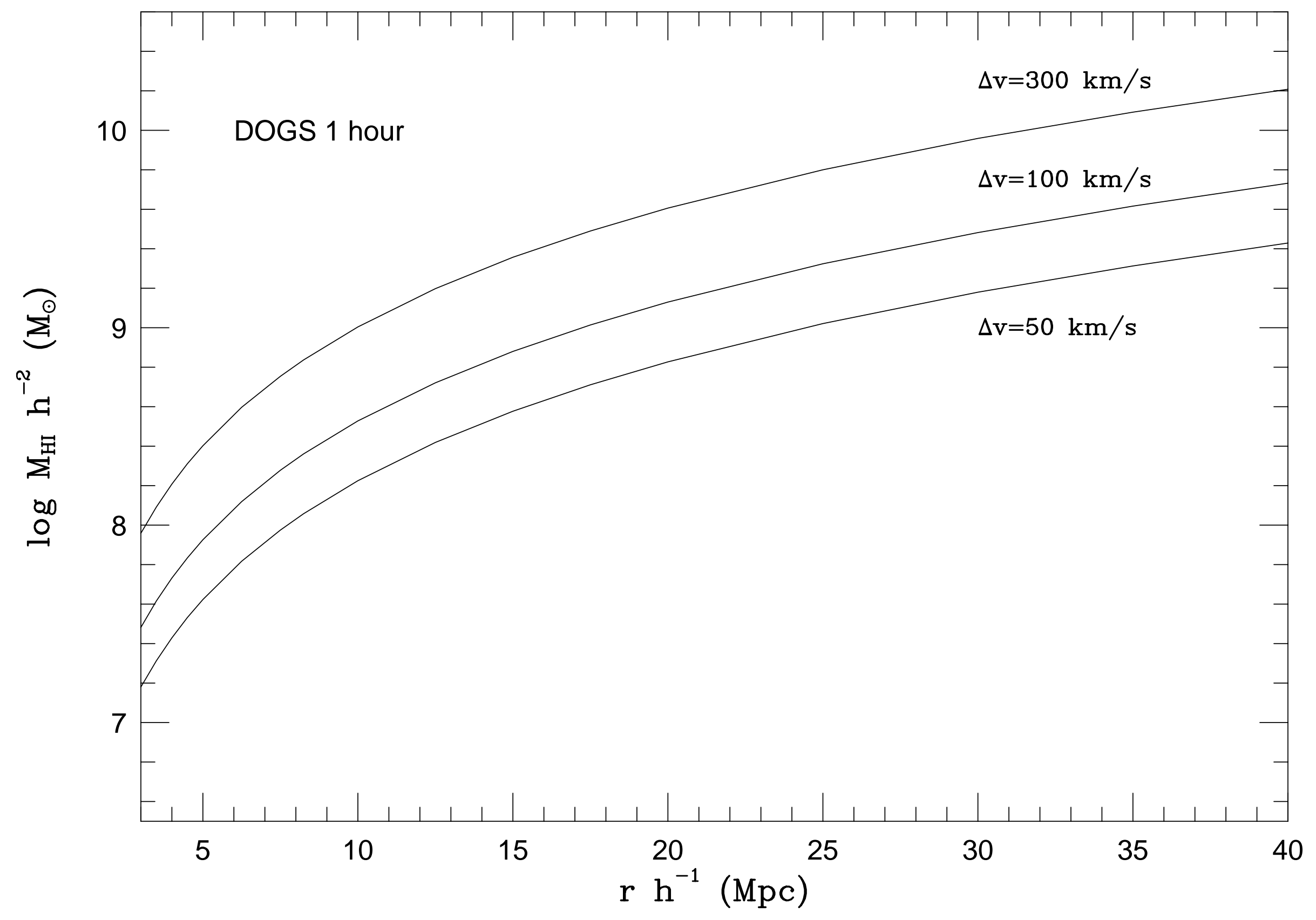




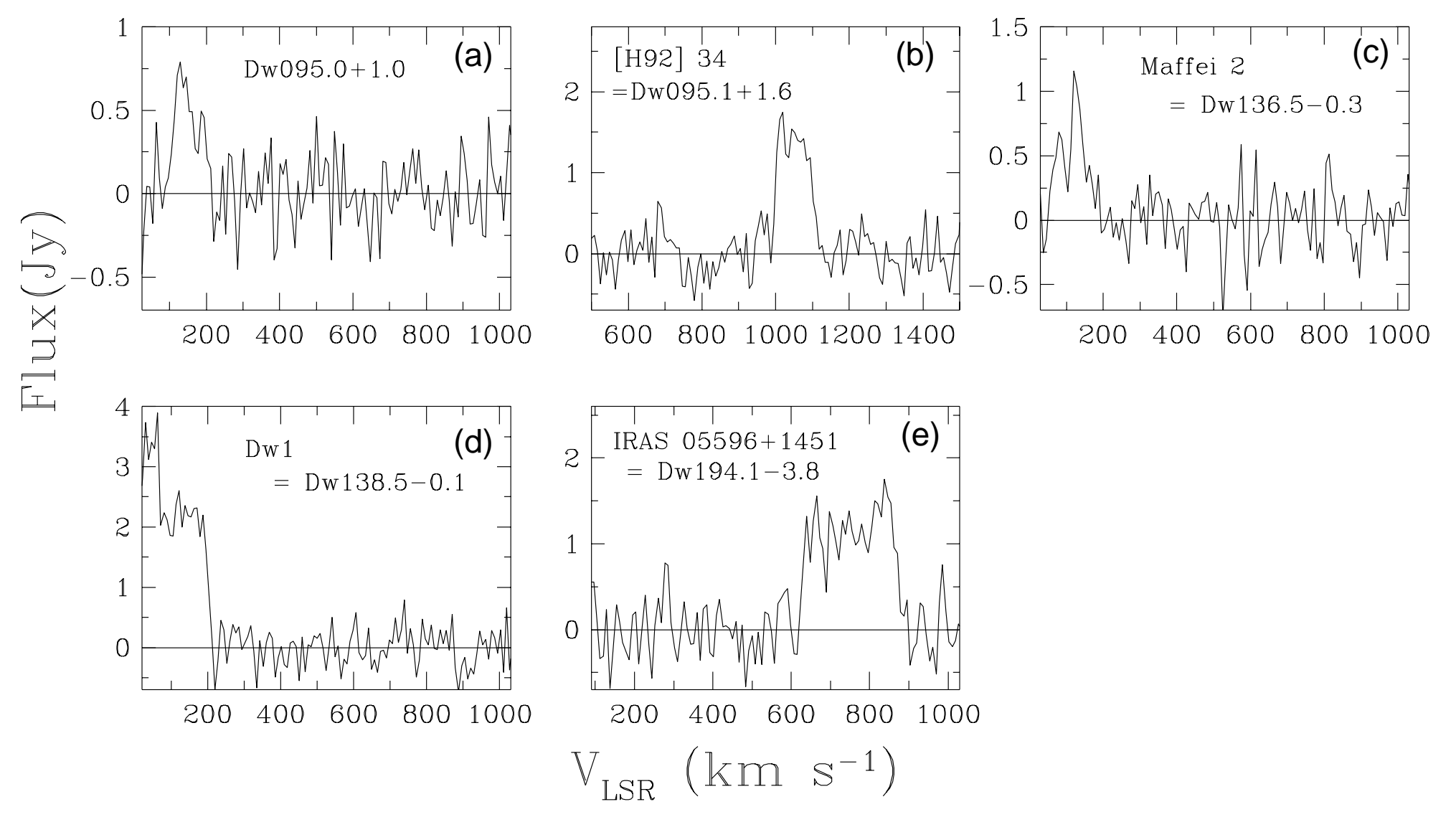




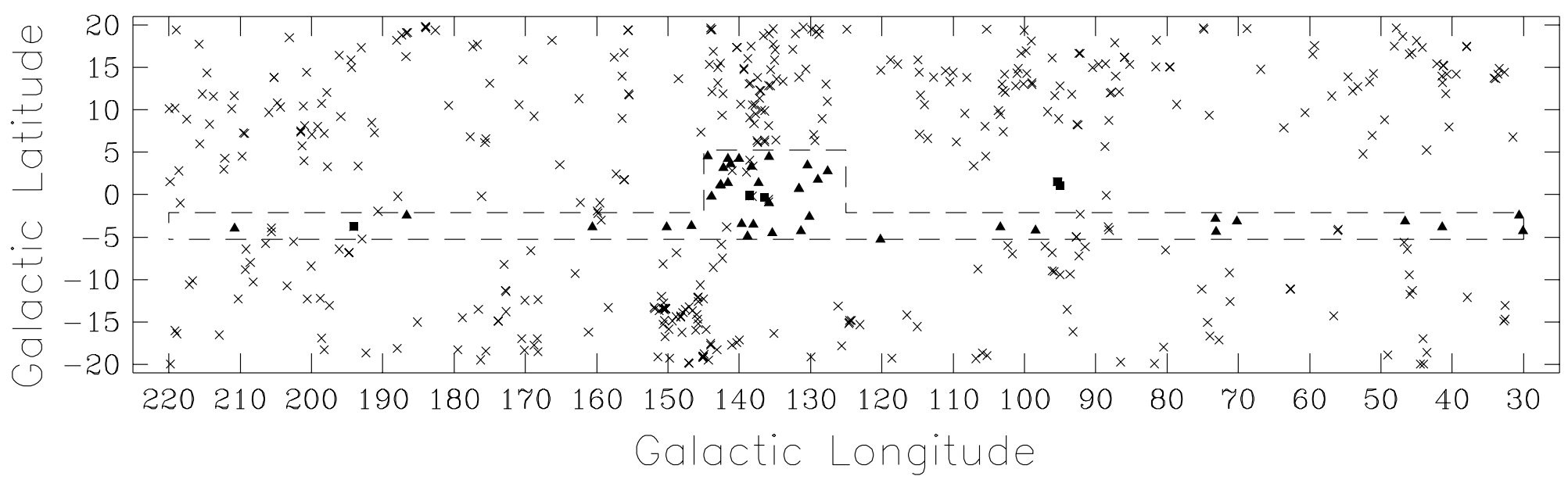

